\title{
Culture and Characteristics of Helicobacter bizzozeronii, a New Canine Gastric Helicobacter sp.
}

\author{
MARJA-LIISA HÄNNINEN, ${ }^{1 *}$ I. HAPPONEN,${ }^{2}$ S. SAARI, ${ }^{3}$ AND K. JALAVA ${ }^{1}$ \\ Department of Food and Environmental Hygiene, ${ }^{1}$ Department of Veterinary Clinical Sciences, ${ }^{2}$ and Institute of \\ Pathology, ${ }^{3}$ Faculty of Veterinary Medicine, University of Helsinki, 00581 Helsinki, Finland
}

\begin{abstract}
Organisms whose cells were large, tight spirals were isolated from gastric biopsies of dogs. Touch cytology samples from all of the dogs contained large spiral organisms. Characteristics of 10 strains are described. These organisms were 5 to $10 \mu \mathrm{m}$ long by $0.3 \mu \mathrm{m}$ wide, and each cell had 10 to 20 sheathed flagella at both ends of the cell. The cells did not have periplasmic fibrils. These organisms were microaerophilic and grew at 37 and $42^{\circ} \mathrm{C}$ but not at $25^{\circ} \mathrm{C}$ on brain heart infusion agar containing blood. They did not grow on brucella blood agar. They were catalase and oxidase positive, hydrolyzed urea but not hippurate, reduced nitrate, and were resistant to nalidixic acid but susceptible to cephalothin and metronidazole. In contrast to Helicobacter felis, they hydrolyzed indoxyl acetate. The sodium dodecyl sulfate-polyacrylamide gel electrophoresis protein profiles of all of the strains were similar, and the protein patterns of these organisms differed from those of other Helicobacter spp. Dot blot DNA-DNA hybridization experiments revealed that the new strains were closely related to each other but clearly different from $\mathrm{H}$. felis, Helicobacter pylori, Helicobacter mustelae, and Campylobacter jejuni. The name Helicobacter bizzozeronii sp. nov. is proposed for these organisms. Our results suggest that other "uncultured" gastric helicobacters may be cultured if optimal culture conditions are found.
\end{abstract}

Since the end of the 19th century several authors have described spiral organisms that occur in the gastric mucosae of dogs and cats $(1,23,25)$. Salomon found that such organisms colonize both the fundus and antrum areas of dogs, and he was able to infect mice with the organisms by feeding them pieces of gastric biopsies (25). The results of the electron microscopic studies of Weber and Schmittdiel (31) confirmed the spiral morphology of the organisms. Lockard and Boler (16) described three morphologically different types of spiral organisms that they found in gastric mucosae of dogs. Structurally, each cell of one of these organisms was a straight cylinder with a filament tightly coiled around its body. The cells of another type were large spirals with coiled helical filaments visible at the crest of each spiral. The cells of the third morphological type were tightly coiled and did not have visible filaments. Lockard and Boler (16) suggested that the three morphological forms represented various stages that occurred during movement of the same organism, and they suggested that the organism should be called Spirochaetales instead of Spirillum rappini, as suggested previously by Rappin (2a, 24). The organisms could not be cultured.

Helicobacter pylori, a human-adapted gastric spiral organism, was cultured for the first time from a gastric biopsy about 10 years ago (18). During the last 10 years, there has been increasing interest in spiral organisms which colonize the gastrointestinal tracts of humans and animals $(2,3,12,13)$. The most important reason for the interest in gastrointestinal spiral organisms is the evident association of chronic $H$. pylori infections of humans with peptic ulceration and gastric cancer $(2$, 20).

In 1988 Lee et al. (14) cultured one of the spiral organisms that had been described morphologically previously from gastric biopsies of dogs and cats. This organism was later assigned to the genus Helicobacter and was named Helicobacter felis

\footnotetext{
* Corresponding author. Mailing address: Department of Food and Environmental Hygiene, Faculty of Veterinary Medicine, University of Helsinki, P.O. Box 6, 00581 Helsinki, Finland. Phone: 358-0-70849704. Fax: 358-0-70849718.
}

(21). H. felis is a long spiral organism, and it has typical periplasmic fibrils (21). The other spiral organism which resembles $H$. felis morphologically when light microscopy is used is also commonly found in gastric mucosae of dogs and cats. If electron microscopy is used, this organism can be differentiated from $H$. felis; the cells are more tightly coiled, and no periplasmic fibrils can be seen $(3,12,16,21)$. Attempts to culture this gastric spiral organism have been unsuccessful (3, 13). Both of these organisms have sheathed flagella at both ends of each cell $(12,21)$. Large, uncultured, morphologically similar organisms found in gastric mucosae of dogs, cats, pigs, and humans have been provisionally placed in the genus "Gastrospirillum" $(10,19,23,27)$.

A large, spiral organism has been found in a minority of human gastric biopsies in association with gastritis $(10,19,32)$. Because this organism has not been cultured so far, its taxonomic description is based on the results of cloning and sequencing of its 16S rRNA gene after PCR amplification of samples obtained from infected gastric mucosae of mice fed gastric mucosa samples from two infected humans (27). The results of these studies suggested that there were probably two species of large spiral organisms ("Gastrospirillum" species 1 and 2) in the human gastric samples because there were two $16 \mathrm{~S}$ rRNA sequences that differed by $3.5 \%$. The $16 \mathrm{~S}$ rRNA sequences obtained were most closely related to the $16 \mathrm{~S}$ rRNA sequence of $H$. felis, and the organisms actually belonged to the genus Helicobacter ("Helicobacter heilmannii"). Human infections with large spiral organisms are thought to be zoonotic because the organisms found in gastric mucosae of humans and dogs are morphologically similar. Also, the results of epidemiological studies have suggested that infections may be transmitted from dogs to humans (27-29).

In this paper we describe the characteristics of large spiral organisms which we cultured from gastric mucosae of dogs. The results of electron microscopic studies, biochemical characteristics, and sodium dodecyl sulfate (SDS)-polyacrylamide gel electrophoresis (PAGE) protein profiles clearly showed that these organisms differed from H. felis, "Flexispira rappini," and other previously cultured Helicobacter spp. that occur in gastric mucosae. The results of DNA-DNA hybridization stud- 
ies confirmed that all 10 strains which we studied were closely related and that they differed from $H$. felis and other related helicobacters. We propose the name Helicobacter bizzozeronii sp. nov. for these organisms. Below we use the name $H$. bizzozeronii for the large, canine, gastric, spiral organism which we studied.

\section{MATERIALS AND METHODS}

Culture of samples. Helicobacters were isolated from biopsy samples obtained from pet dogs after endoscopy or from gastric mucosae of euthanized dogs postmortem at the University of Veterinary Medicine in Helsinki, Finland. The samples were obtained from the antrum and fundus areas. The biopsy samples either were cultured directly within $1 \mathrm{~h}$ of sampling or were stored in Portagerm pylori transport medium (BioḾerieux SA, Marcy l'Etoile, France) for 2 to $12 \mathrm{~h}$ before they were cultured. Direct Gram stain (mucus touch cytology) and rapid urease tests were performed with the biopsy samples, and histological samples were fixed with $10 \%$ formaldehyde for later analysis.

The samples were crushed in 300 - to 500 - $\mu$ l portions of brain heart infusion (BHI) (Difco) broth containing 7\% horse serum, and the preparations were spread onto fresh BHI blood agar plates containing antibiotics $(2.5 \mu \mathrm{g}$ of trimethoprim per $\mathrm{ml}, 5 \mu \mathrm{g}$ of vancomycin per $\mathrm{ml}, 1.25 \mathrm{IU}$ of polymyxin $\mathrm{B}$ per $\mathrm{ml}$, and $2 \mu \mathrm{g}$ of amphotericin $\mathrm{B}$ per $\mathrm{ml}$ ). These plates were incubated under microaerophilic conditions by using a model BR 56 gas-generating kit (Oxoid, Basingstoke, United Kingdom) with the lids uppermost at $37^{\circ} \mathrm{C}$ for 10 days. Usually growth occurred after 3 to 5 days of incubation as a thin spreading film. The method used for primary identification has been described previously (9). The isolates that had the typical spiral morphology were stored in sterile skim milk at $-70^{\circ} \mathrm{C}$. In this paper we describe the phenotypic and genotypic characteristics of $10 \mathrm{H}$. bizzozeronii strains that were isolated from different dogs with various degrees of gastritis between 1989 and 1994.

We used a human Campylobacter jejuni strain, H. felis CS1 (= ATCC 49179), H. pylori 26695 and TX 30A, Helicobacter mustelae ATCC 43772, and "F. rappini", (a dog isolate) as controls.

Ultrastructural studies. The culture samples used for scanning electron microscopy with a JEOL model JMS 820 scanning electron microscope were filtered nylon membranes (pore size, $0.45 \mu \mathrm{m}$ ), serially dehydrated in ethanol, critical point dried (Balzer's CPD 020), mounted on brass studs, and coated with gold by using a JEOL model JFC 1100 sputter coater (17). For transmission electron microscopy bacteria were resuspended in physiological $\mathrm{NaCl}$ and a turbid suspension was stained with $1 \%$ phosphotungstic acid and examined with a JEOL model 1200 EX electron microscope.

The histological samples used for transmission electron microscopy were fixed in $2.5 \%$ glutaraldehyde in Sörensen buffer containing $0.1 \mathrm{mmol}$ of phosphate per liter ( $\mathrm{pH} \mathrm{7.3).} \mathrm{After} \mathrm{dehydration} \mathrm{in} \mathrm{acetone,} \mathrm{the} \mathrm{samples} \mathrm{were} \mathrm{embedded} \mathrm{in}$ Epon. Thin sections were stained with uranyl acetate and lead citrate.

Biochemical and tolerance tests. The commonly used phenotypic tests recommended by Ursing et al. (30) for description of new Campylobacter species and by Goodwin and Armstrong for $H$. pylori studies (5) were used to characterize the isolates which we studied. The strains were first tested for oxidase activity, catalase activity (with 3\% hydrogen peroxide), rapid urease activity, and hippurate hydrolysis. All of the strains were also tested by using API Campy test strips (Biomerieux SA) and Rosco diagnostic tablets (Rosco Diagnostica, Taastrup, Denmark). The tests used included tests for urease activity; nitrate reductase activity; indoxyl acetate hydrolysis; hippurate hydrolysis; triphenyltetrazolium chloride (TTC) reduction; gamma-glutamyl transpeptidase activity, pyrrolidonyl, L-arginine, and L-aspartate arylamidase activities; and alkaline phosphatase activity. A DNase production test was also performed (15).

The following tests were performed on BHI blood agar: tests for tolerance to $1 \%$ glycine, $1 \%$ ox bile (Difco), and $2.5 \% \mathrm{NaCl}$. Antibiotic susceptibility tests were performed by using $\mathrm{BHI}$ blood agar and nalidixic acid disks $(30 \mu \mathrm{g} ; \mathrm{AB}$ Biodisk, Solna, Sweden), cephalothin disks ( $30 \mu \mathrm{g}$ : AB Biodisk), metronidazole disks $(50 \mu \mathrm{g}$; Rosco), and cefoperazone disks (60 $\mu \mathrm{g}$; Rosco). All growth and tolerance test preparations were incubated for 7 days in a microaerophilic atmosphere at $37^{\circ} \mathrm{C}$.

Growth conditions. We tested growth of the organisms on BHI blood agar, brucella blood agar, nutrient blood agar, and Muller-Hinton blood agar. Growth at $25,30,37$, and $42^{\circ} \mathrm{C}$ was determined by using BHI blood agar. Growth in a shallow liquid medium was determined by using BHI broth containing $10 \%$ horse serum, and during incubation the medium was shaken at $100 \mathrm{rpm}$. The cultures were incubated for 7 days in a microaerophilic atmosphere at $37^{\circ} \mathrm{C}$.

SDS-PAGE protein profiles. The organisms were grown on BHI blood agar plates for 3 to 5 days, and then the growth was collected and washed with phosphate-buffered saline. Samples were diluted in sample buffer and electrophoresed with a Mini Protean II electrophoresis cell (Bio-Rad Laboratories, Richmond, Calif.) by using $10 \%$ separation gels (11). The gels were stained with Coomassie blue.

DNA-DNA hybridization. Chromosomal DNA was isolated by the modified method of Pitcher et al. (22). Phenol-chloroform was used for DNA extraction instead of chloroform, and an RNase treatment was used to remove the RNA.
DNA-DNA hybridization experiments were performed by using a dot blot assay, nylon membranes (Hybond N; Amersham, Amersham, United Kingdom), and a vacuum blotting apparatus (Pharmacia LKB Biotechnology, Sollentuna, Sweden). Aliquots (50, 5 , and $0.5 \mathrm{ng}$ ) of diluted chromosomal DNAs were added to the nylon membranes. The control organisms used were Escherichia coli, C. jejuni, $H$. felis CS1 (= ATCC 49179), H. pylori TX 30A, and H. mustelae ATCC 43772. The probes used were the genomic DNAs from two H. bizzozeronii strains isolated from dogs, $H$. felis CS1, and a " $F$. rappini" strain isolated from a dog. The probes were labelled by random priming with digoxigenin-dUTP according to the manufacturer's instructions for the DNA labelling and detection kit (Boehringer, Mannheim, Germany). Dot blot hybridization preparations containing digoxigenin-labelled probes were incubated at $68^{\circ} \mathrm{C}$ overnight. After hybridization, the membranes were washed twice $(5 \mathrm{~min}$ each in $2 \times \mathrm{SSC}[1 \times \mathrm{SSC}$ is $0.15 \mathrm{M} \mathrm{NaCl}$ plus $0.015 \mathrm{M}$ sodium citrate]) containing $0.1 \%$ SDS at room temperature and then twice $\left(15 \mathrm{~min}\right.$ each) in the same buffer at $68^{\circ} \mathrm{C}$. The results were determined visually on the basis of the intensity of $\mathrm{AP}$ color that developed with different dilutions of DNA. Four identical membranes that included the control and study strains were produced, and these membranes were probed with all of the probe strains representing different Helicobacter spp. at the same time. The tests were repeated seven times.

\section{RESULTS}

Ultrastructure. Our ultrastructure studies revealed that the organisms which we studied were similar to the organisms found previously in gastric mucosae of dogs $(12,16)$ and resembled an organism called " $H$. heilmannii" found in human gastric biopsy samples (10). The cells were large spirals that were 5 to $10 \mu \mathrm{m}$ long and $0.3 \mu \mathrm{m}$ wide and had three to eight spirals per cell and no periplasmic fibrils (Fig. 1A and B). There were multiple sheathed flagella at both ends of each cell (Fig. 1B); these flagella were slightly off center at the ends of the cells (Fig. 1C). Coccoid forms were observed in older cultures (Fig. 1A). The ultrastructural characteristics of the organisms were examined several times after several subcultures and were the same in all studies.

The typical in vivo location of $H$. bizzozeronii in a gastric gland is shown Fig. 1D.

Isolation and growth characteristics. Most of the strains $(8$ of 10 strains) were isolated from corpus samples. In two cases identical strains were isolated from corpus and antrum samples. We studied 30 dogs, and large spiral or helical organisms were found in all touch cytology samples. $H$. bizzozeronii was isolated from $10 \mathrm{dogs}$, and $H$. felis was isolated from two dogs. Differentiation between $H$. bizzozeronii and $H$. felis was originally based on the results of ultrastructural studies. During primary isolation the organisms grew after 5 to 10 days of incubation as thin, spreading nonhemolytic areas on moist BHI blood agar. In subsequent cultures the incubation time necessary for growth to become visible was between 3 and 5 days. Usually, $H$. felis exhibited visible growth after a shorter incubation time than $H$. bizzozeronii.

Growth on different media and conditions. The best growth was obtained on fresh BHI blood agar. The organisms grew also on Mueller-Hinton agar and nutrient agar supplemented with blood or horse serum. There was minimal growth on brucella blood agar. Only slight growth occurred in broth media, even though serum was added and a shallow medium was used (5).

Biochemical and tolerance tests. A total of 24 tests were performed in order to study the characteristics of $10 \mathrm{H}$. bizzozeronii isolates obtained from dog gastric mucosae. Typical biochemical characteristics and the results of the tolerance tests are shown in Table 1 . All of the strains were oxidase, catalase, and urease positive. They reduced nitrate and TTC, they were positive in the indoxyl acetate, gamma-glutamyl transpeptidase, and alkaline phosphatase tests, and they were negative in the hippurate hydrolysis, pyrrolidonyl arylamidase, L-arginine arylamidase, and L-aspartate arylamidase tests. They were resistant to nalidixic acid and susceptible to cephalothin, ce- 

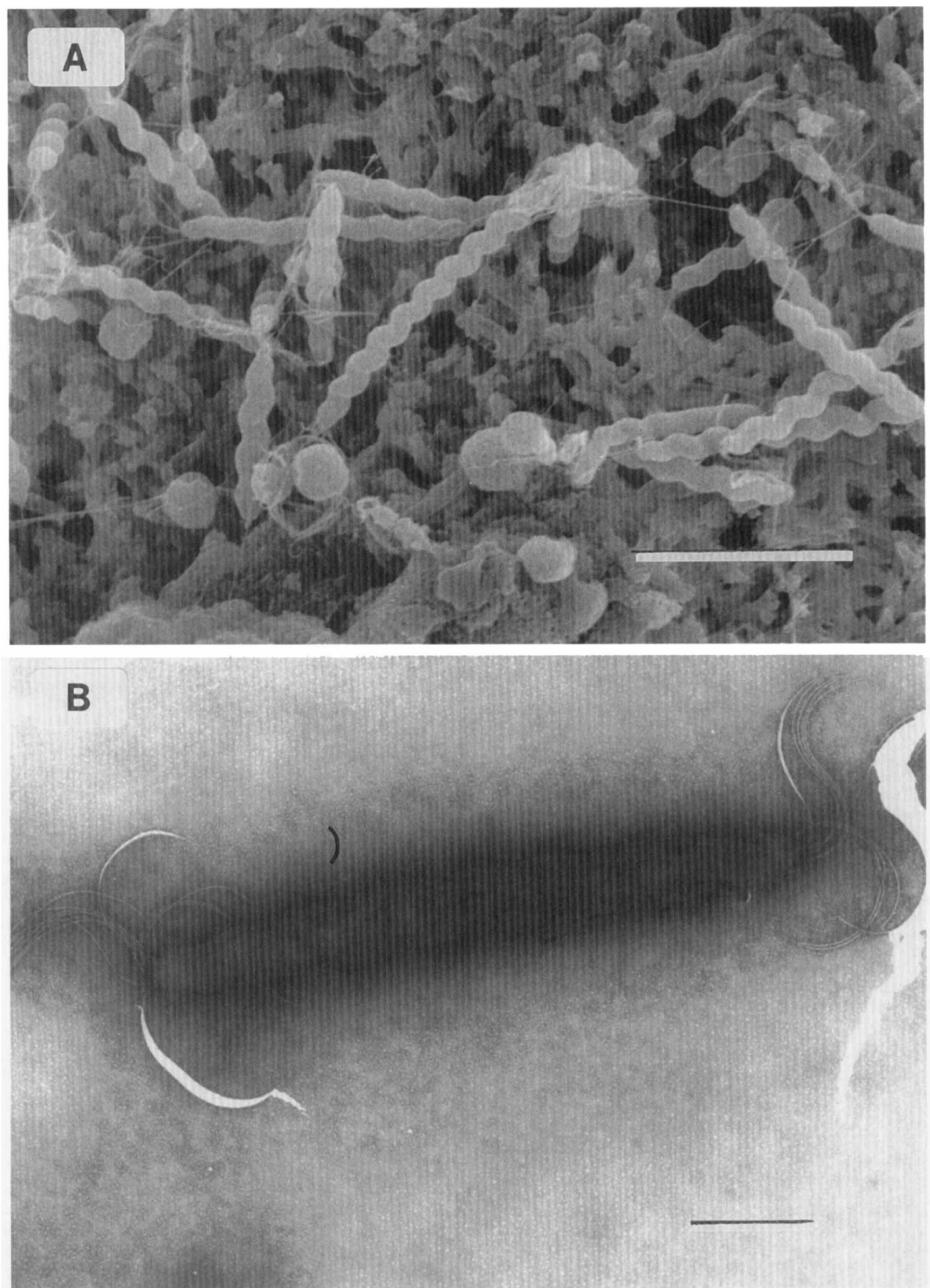

FIG. 1. (A) Scanning electron micrograph of $H$. bizzozeronii sp. nov, on a nylon membrane after growth on BHI blood agar, showing the characteristic spiral morphology. No periplasmic fibrils are visible. Coccoid forms are evident. Bar $=5 \mu \mathrm{m}$. (B) Transmission electron micrograph of a negatively stained cell of $H$. bizzozeronii sp. nov showing the spiral morphology and the multiple sheathed flagella at both ends of the cell. Bar $=0.5 \mu \mathrm{m}$. (C) Transmission electron micrograph of a negatively stained $H$. bizzozeronii sp. nov. cell, showing the position of flagella slightly off center. Bar $=0.5 \mu \mathrm{m}$. (D) Presence of $H$. bizzozeronii sp. nov. in vivo in the gastric gland lumen of a dog. Abbreviations: $\mathrm{P}$, parietal cell; $\mathrm{C}$, chief cell. The arrow indicates sheathed flagella. Bar $=1 \mu \mathrm{m}$. 

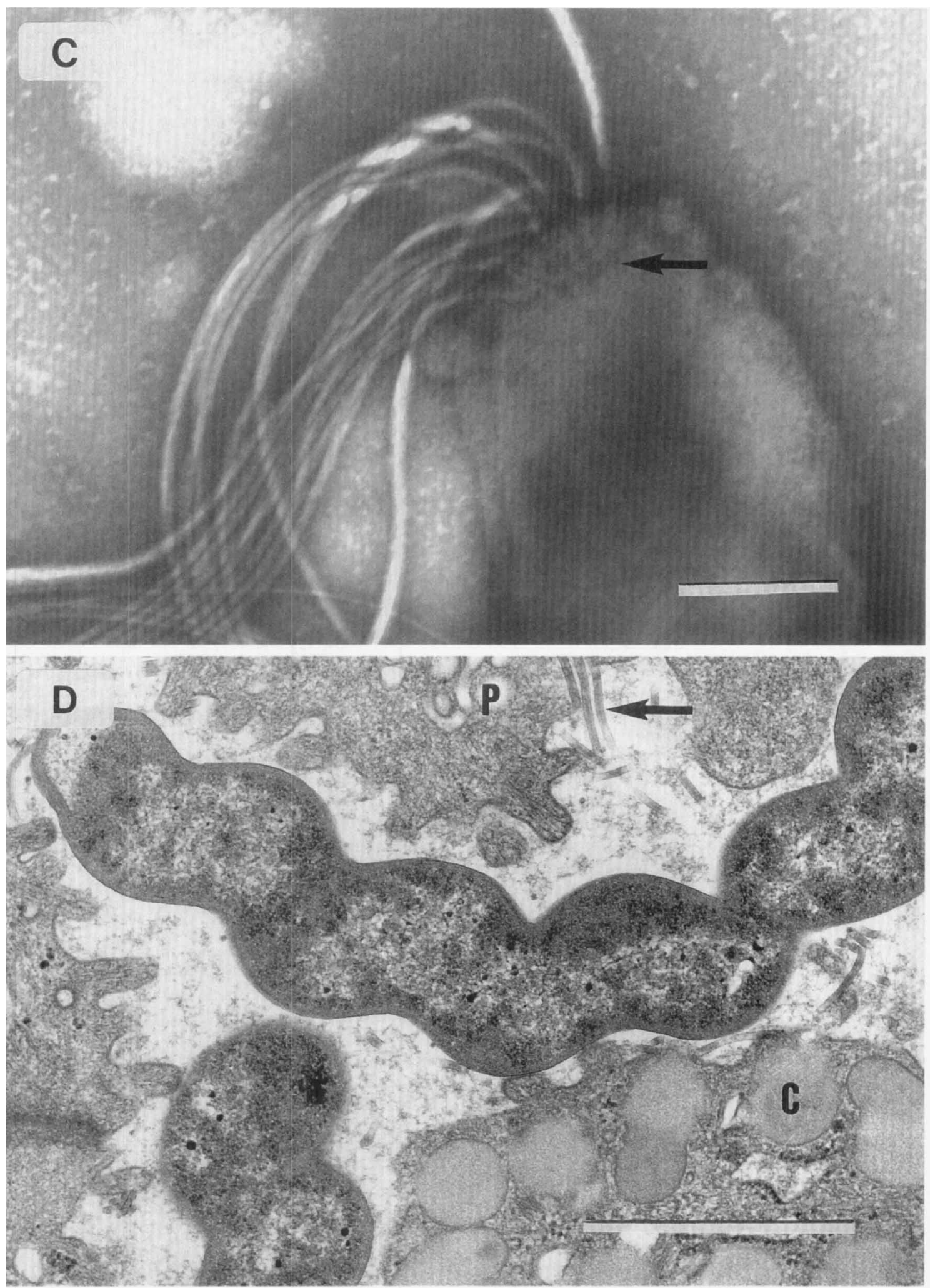

foperazone, and metronidazole. They did not grow on medium containing $1 \%$ ox bile, $1 \%$ glycine, or $1.5 \% \mathrm{NaCl}$. They grew at 37 and $42^{\circ} \mathrm{C}$ but not at $25^{\circ} \mathrm{C}$. All of the characteristics studied except indoxyl acetate hydrolysis were similar to the charac- teristics found for $H$. felis CS1. All $H$. bizzozeronii strains and H. felis CS1 produced DNase.

SDS-PAGE protein profile patterns. The total-protein profile patterns of the $H$. bizzozeronii strains were similar (Fig. 2). 
TABLE 1. Characteristics of $H$. bizzozeronii and related gastric helicobacters ${ }^{a}$

\begin{tabular}{|c|c|c|c|c|c|}
\hline Characteristic & $\begin{array}{l}\text { Helicobacter } \\
\text { bizzozeronii }\end{array}$ & $\begin{array}{l}\text { Helicobacter } \\
\quad \text { felis }\end{array}$ & $\begin{array}{c}\text { Helicobacter } \\
\text { pylori }\end{array}$ & $\begin{array}{l}\text { Helicobacter } \\
\text { acinonyx }\end{array}$ & $\begin{array}{l}\text { "Flexispira } \\
\text { rappini" }\end{array}$ \\
\hline Periplasmic fibrils & - & + & - & + & + \\
\hline Location of flagella & Bipolar & Bipolar & Polar & Polar & Bipolar \\
\hline No. of flagella & $10-20$ & $14-20$ & $4-8$ & $2-5$ & $10-20$ \\
\hline Flagellar sheath & + & + & + & + & + \\
\hline Oxidase activity & $+(10 / 10)^{b}$ & + & + & + & + \\
\hline Catalase activity & $+(10 / 10)$ & + & + & + & + \\
\hline Urease activity (rapid) & $+(10 / 10)$ & + & + & + & + \\
\hline Nitrate reduction & $+(10 / 10)$ & + & - & - & - \\
\hline Hippurate hydrolysis & $-(10 / 10)$ & - & - & - & - \\
\hline Indoxyl acetate hydrolysis & $+(10 / 10)$ & - & - & - & ND \\
\hline$\gamma$-Glutamyl transpeptidase activity & $+(10 / 10)$ & + & + & + & + \\
\hline TTC reduction & $+(10 / 10)$ & - & + & ND & ND \\
\hline Alkaline phosphatase activity & $+(10 / 10)$ & + & + & + & + \\
\hline DNase activity & $+(10 / 10)$ & + & + & ND & ND \\
\hline \multicolumn{6}{|l|}{ Growth at: } \\
\hline $25^{\circ} \mathrm{C}$ & $-(10 / 10)$ & - & - & - & \\
\hline $37^{\circ} \mathrm{C}$ & $+(10 / 10)$ & + & + & + & \\
\hline $42^{\circ} \mathrm{C}$ & $+(10 / 10)$ & - & - & + & \\
\hline Tolerance to $1 \%$ ox bile & $-(10 / 10)$ & - & - & - & \\
\hline \multicolumn{6}{|l|}{ Susceptibility to: } \\
\hline Nalidixic acid $(30 \mu g$-disk $)$ & $\mathrm{R}(10 / 10) \mathrm{R}$ & $\mathbf{R}$ & $\mathbf{R}$ & $\mathrm{R}$ & \\
\hline Cephalothin ( $30 \mu$ g-disk) & $\mathrm{S}(10 / 10) \mathrm{S}$ & $\mathrm{S}$ & $\mathrm{s}$ & $\mathrm{R}$ & \\
\hline Cefoperazone (60- $\mu \mathrm{g}$ disk) & $S(10 / 10) S$ & $\mathrm{~S}$ & ND & ND & \\
\hline Metronidazole (50- $\mu \mathrm{g}$ disk $)$ & $\mathrm{S}(8 / 10) \mathrm{S}$ & S & $\mathrm{S}$ & $\mathrm{S}$ & \\
\hline
\end{tabular}

${ }^{a}-$, negative or not present; + , positive or present; ND, not determined; $\mathrm{R}$, resistant; $\mathrm{S}$, susceptible.

${ }^{b}$ The data in parentheses are number of strains that have the characteristic indicated/number of strains tested.

The major bands were bands at 30 and $42 \mathrm{kDa}$, a double band at 50 to $55 \mathrm{kDa}$, bands at 59 and $62 \mathrm{kDa}$, and two double bands at 72 to 74 and 84 to $88 \mathrm{kDa}$. In contrast, the protein profile of $H$. felis CS1 differed from the H. bizzozeronii profiles. H. felis produced major bands at $40,53,56,59,66,72$, and $105 \mathrm{kDa}$. In particular, the strong bands at 56 and $105 \mathrm{kDa}$ found in the $H$. felis profile were not present in the $H$. bizzozeronii profile. The bands at 62 and $66 \mathrm{kDa}$ represented urease B subunits in $H$. bizzozeronii and $\mathrm{H}$. felis, respectively (7). The intensities of the bands at 59,62, and $66 \mathrm{kDa}$ varied according to the age of the culture. In young cultures (cultures that were 3 to 5 days old) the band at $59 \mathrm{kDa}$ was strong, and in cultures that were 5 to 10 days old the urease B subunit bands predominated.

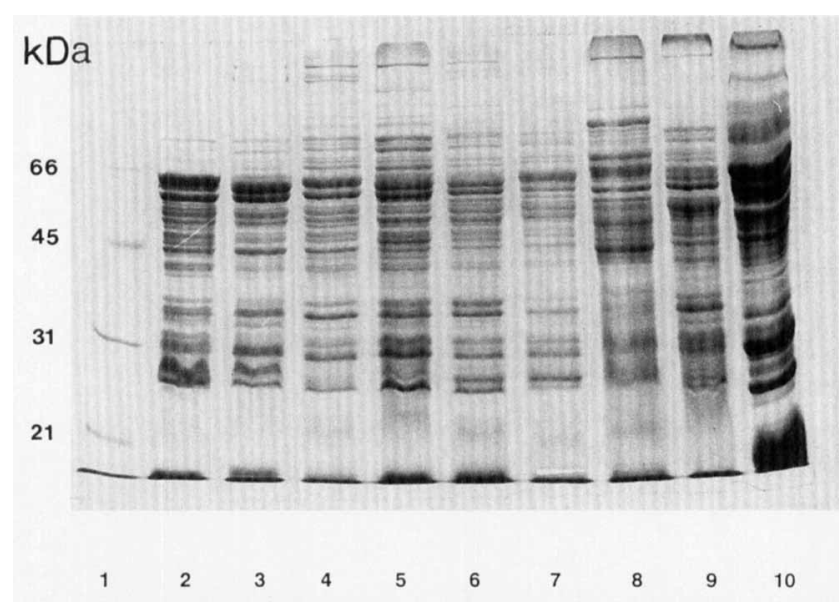

FIG. 2. SDS-PAGE protein profiles (on a $10 \%$ separation gel) of $\mathrm{H}$. bizzozeronii sp. nov, and related species. Lane 1, molecular weight markers (molecular weights, 21,500, 31,000, 45,000, and 66,200); lanes 2 through 7 and $9, H$. bizzozeronii; lane $8, H$. felis CS1; lane $10, H$. pylori.
DNA-DNA hybridization. The relative levels of hybridization were assessed by comparing the intensities of the color that developed with the three concentrations of DNA added to the membrane. The DNA-DNA hybridization results showed that all of the $H$. bizzozeronii strains hybridized intensively with the two $H$. bizzozeronii probe strains used. The probes hybridized weakly with the DNA of $H$. felis CS1. No reaction was observed with $C$. jejuni, $H$. mustelae, or $H$. pylori. The $H$. felis CS1 probe DNA hybridized weakly with the $H$. bizzozeronii strains. " $F$. rappini" did not hybridize with the $H$. bizzozeronii strains.

\section{DISCUSSION}

The number of new species in the genus Helicobacter has expanded greatly since the first two species, $H$. pylori and $H$. mustelae (6), were described. Twelve Helicobacter species have been described in recent papers, and at least six new Helicobacter species are waiting for more definite taxonomic descriptions (4). In this paper we describe a new Helicobacter species which has been studied ultrastructurally by using histological tissue samples from dogs for more than 100 years but which has not been cultured previously $(2 \mathrm{a}, 12,16,24)$. The morphology of this bacterium is similar to the morphology of organisms provisionally called " $H$. heilmannii" and "Gastrospirillum suis" and found in histological specimens from humans $(10,19)$ and pigs (23), respectively. $H$. bizzozeronii is a 5 - to 10 - $\mu$ m-long, spiral bacterium that has multiple sheathed flagella at both ends of each cell. The most significant morphological difference between this organism and $H$. felis is the lack of periplasmic fibrils in the former. When light microscopy is used, reliable differentiation of $H$. bizzozeronii and $H$. felis is not possible because both organisms have a spiral morphology and their sizes are similar. Although $H$. bizzozeronii was more common than $H$. felis in gastric biopsy samples from dogs, $H$. felis was also detected. On BHI blood agar the growth of $H$. bizzozeronii and the growth of $H$. felis were similar. After primary incuba- 
tion, both organisms grew as thin, spreading nonhemolytic films on the agar. Both organisms produced coccoid forms in older cultures, as described previously for campylobacters and other helicobacters (5).

Helicobacter spp. can be divided into two groups on the basis of their primary ecological niches (either the gastric mucosa or the lower gastrointestinal tract) (26). H. bizzozeronii is a species that is adapted to gastric areas; $H$. bizzozeronii cells are found superficially in mucus and in gastric glands, as well as inside parietal cells $(3,12,16)$. All known primary gastric Helicobacter spp., including $H$. bizzozeronii, contain rapidly preformed urease, which probably plays a role during colonization $(2,3,7)$. One other typical characteristic of the gastric helicobacters is their sensitivity to bile and bile salts $(5,8)$ compared with the intestinal helicobacters, which can tolerate bile salts at concentrations of up to $20 \%(4)$. H. bizzozeronii is sensitive to bile.

Other biochemical characteristics of $H$. bizzozeronii, including utilization of glycine, tolerance to $\mathrm{NaCl}$, susceptibility to cephalothin, cefoperazone, and metronidazole, and resistance to nalidixic acid, were similar to characteristics of $H$. felis. Both species also produced DNase. $H$. bizzozeronii differed from $H$. felis by reacting positively in indoxyl acetate hydrolysis and TTC reduction tests; $H$. felis is negative in these tests (21).

Our SDS-PAGE profile analysis of whole-cell proteins produced reliable, reproducible results which could be used to differentiate $H$. bizzozeronii from $H$. felis. $H$. felis produced major protein bands at 56,72 , and $105 \mathrm{kDa}$ which were not found in $H$. bizzozeronii. These proteins might be associated with the periplasmic fibrils observed on the surfaces of $H$. felis cells. The ureases of helicobacters are visible on denatured gels as two subunits, one at about 62 to $66 \mathrm{kDa}$ and one at $30 \mathrm{kDa}$ (7). The $H$. felis urease had two subunits, which had molecular weights of about 66,000 and 30,000 (7). The molecular weight of the putative $H$. bizzozeronii urease B subunit $(62,000$ to 63,000 ) was lower than the molecular weight of the urease B subunit of $H$. felis. A more detailed study of the membrane proteins of canine gastric helicobacters is in progress.

Our DNA-DNA hybridization data confirmed that all of the large, canine, spiral organisms were closely related to each other and were clearly different from $H$. pylori and $H$. mustelae. There was some hybridization between $H$. bizzozeronii and $H$. felis strains; a weak color developed when $H$. felis DNA was hybridized with two different probe DNAs from canine helicobacters or when the DNAs of the 10 canine $H$. bizzozeronii strains were hybridized with the $H$. felis probe DNA.

Because $H$. bizzozeronii has not been cultured previously, there have been suggestions that $H$. felis and $H$. bizzozeronii may represent different forms of the same organism $(16,27)$. Our data confirmed that these two large, gastric, spiral organisms differ morphologically, phenotypically, and genetically and that they are two different Helicobacter spp. which both colonize the gastric mucosae of dogs.

It was not possible to study the relationship between the human parasite " $H$. heilmannii" and the canine parasite $H$. bizzozeronii because " $H$. heilmannii" has not been cultured yet. Morphological and 16S rRNA sequence data are the only data for " $H$. heilmannii" that are available. Morphologically, the canine and human organisms are similar. A $16 \mathrm{~S}$ rRNA sequence analysis of canine $H$. bizzozeronii strains is in progress in our laboratory. A comparison of the $16 \mathrm{~S}$ rRNA sequences should provide more information concerning the possible relationship between the canine and human organisms. However, in order to confirm the possible identity of $H$. bizzozeronii and " $H$. heilmannii," it will be necessary to compare phenotypic and genetic characteristics of cultured organisms.

Taxonomic description of Helicobacter bizzozeronii sp. nov.
Helicobacter bizzozeronii (biz.zo'zero.ni.i. L. gen. n. bizzozeronii, of Bizzozero, referring to Guilio Bizzozero, an Italian pathologist who was one of the first scientists to describe canine gastric spiral organisms [1]). The cells are spirals that are 0.3 by 5 to $10 \mu \mathrm{m}$. They do not have periplasmic fibrils. In older cultures coccoid forms predominate. The cells are gram negative and nonsporulating. They are motile by means of tufts of 10 to 20 sheathed flagella at both ends of each cell. Individual colonies are not usually produced on agar media, but cultures grow as spreading films on fresh moist agar media. All strains are oxidase, catalase, and urease positive. They reduce nitrate and TTC, and they are positive in indoxyl acetate, gammaglutamyl transpeptidase, and alkaline phosphatase tests. They are negative in hippurate hydrolysis, pyrrolidonyl arylamidase, L-arginine arylamidase, and L-aspartate arylamidase tests. They are resistant to nalidixic acid and susceptible to cephalothin, cefoperazone, and metronidazole. They do not grow on medium containing $1 \%$ ox bile, $1 \%$ glycine, or $1.5 \% \mathrm{NaCl}$. They grow at 37 and $42^{\circ} \mathrm{C}$ but not at $25^{\circ} \mathrm{C}$. All of the biochemical and tolerance characteristics which we studied except indoxyl acetate hydrolysis are similar to the characteristics of $H$. felis CS1. All $H$. bizzozeronii strains and $H$. felis CS1 produce DNase. Two strains have been deposited in the culture collection of the University of Göteborg, Sweden: CCUG 35045 and CCUG 35046.

\section{REFERENCES}

1. Bizzozero, G. 1893. Sulle ghiandole tubulari del tubo gastroenterico e sui rapporti del loro coll'epitelio de rivestimento della mucosa. Atti R. Accad. Sci. Torino 28:233-251.

2. Blaser, M. J. 1993. Helicobacter pylori: microbiology of a 'slow' bacterial infection. Trends Microbiol. 1:255-260.

2a.Breed, R. S., E. G. D. Murray, and A. P. Hichens. 1948. Family Pseudomonadaceae, p. 217. In Bergey's manual of determinative bacteriology, 6th ed. Williams and Wilkins, Baltimore.

3. Fox, J. G., and A. Lee. 1993. Gastric Helicobacter infection in animals: natural and experimental infections, p. 407-430. In C. S. Goodwin and B. W. Worsley (ed.), Helicobacter pylori: biology and clinical practice. CRC Press, Boca Raton, Fla.

4. Fox, J. G., L. L. Yan, F. E. Dewhirst, B. J. Paster, B. Shames, J. C. Murphy, A. Hayward, J. C. Belcher, and E. N. Mendes. 1995. Helicobacter bilis sp. nov., a novel Helicobacter species isolated from bile, livers, and intestines of aged, inbred mice. J. Clin. Microbiol. 33:445-454.

5. Goodwin, C. S., and J. A. Armstrong. 1990. Microbiological aspects of $\mathrm{He}$ licobacter pylori (Campylobacter pylori). Eur. J. Clin. Microbiol. Infect. Dis. 9:1-13.

6. Goodwin, C. S., J. A. Armstrong, T. Chilvers, M. Peters, M. D. Collins, L. Sly, W. McConnell, and W. E. S. Harper. 1989. Transfer of Campylobacter pylori and Campylobacter mustelae to Helicobacter gen. nov, as Helicobacter pylori comb. nov. and Helicobacter mustelae comb. nov., respectively. Int. J. Syst. Bacteriol. 39:397-405.

7. Gootz, T. D., G. I. Pedez-Perez, J. Clancy, B.-A. Martin, A. Tait-Kamradt, and M. J. Blaser. 1994. Immunological and molecular characterization of Helicobacter felis urease. Infect. Immun. 62:793-798.

8. Hänninen, M.-L. 1991. Sensitivity of Helicobacter pylori to different bile salts. Eur. J. Clin. Microbiol. Infect. Dis. 10:515-518.

9. Hänninen, M.-L., K. Jalava, S. Saari, I. Happonen, and E. Westermarck. 1995. Culture of "Gastrospirillum" from gastric biopsies of dogs. Eur. J. Clin. Miocrobiol. Infect. Dis. 14:145. (Letter.)

10. Heilmann, K. L., and F. Bochard. 1991. Gastritis due to spiral-shaped bacteria other than Helicobacter pylori: clinical, ultrastructural findings. Gut 32:137-140.

11. Laemmli, U. K. 1970 . Cleavage of structural proteins during the assembly of the head of bacteriophage T4. Nature (London) 227:680-685.

12. Lee, A. 1989. Human gastric spirilla other than C. pylori, p. 222-240. In M. J. Blaser (ed.), Campylobacter pylori in gastritis and peptic ulcer disease. IgakuShoin Medical Publishers, New York.

13. Lee, A., J. Fox, and S. L. Hazell. 1993. The pathogenicity of Helicobacter pylori: a perspective. Infect. Immun. 61:1601-1610.

14. Lee, A., S. L. Hazell, J. O'Rourke, and S. Kouprach. 1988. Isolation of a spiral-shaped bacterium from the cat stomach. Infect. Immun. 56:2843-2850.

15. Lior, H., and A. Patel. 1987. Improved toluidine-blue agar for detection of DNA hydrolysis of campylobacters. J. Clin. Microbiol. 25:2030-2031.

16. Lockard, V. G., and R. K. Boler. 1970 . Ultrastructure of a spiralled microorganism in the gastric mucosa of dogs. Am. J. Vet. Res. 31:1453-1462.

17. Lounatmaa, K. 1985. Electron microscopic methods for the study of bacte- 
rial surface structures, p. 695-698. In T. K. Korhonen, E. A. Dawes, and P. H. Mäkelä (ed.), Enterobacterial surface antigens: methods for molecular characterization. Elsevier, Amsterdam.

18. Marshall, B. J., and J. R. Warren. 1984. Unidentified curved bacilli in the stomach of patients with gastritis and peptic ulceration. Lancet i:1311-1314.

19. McNulty, C. A. M., J. C. Dent, and A. Carry. 1989. New spiral bacterium in gastric mucosa. J. Clin. Pathol, 42:585-591.

20. Nomura, A., G. N. Stemmermann, P. Chyou, G. I. Perez-Perez, and M. J. Blaser. 1994. Helicobacter pylori and the risk for duodenal and gastric ulceration. Ann. Intern. Med. 120:977-981.

21. Paster, B. J., A. Lee, J. G. Fox, F. E. Dewhirst, L. A. Tordoff, G. J. Fraser, J. R. O'Rourke, N. S. Taylor, and R. Ferrero. 1991. Phylogeny of Helicobacter felis sp. nov., Helicobacter mustelae, and related bacteria. Int. J. Syst. Bacteriol. 41:31-38.

22. Pitcher, D. G., N. A. Sauders, and R. J. Owen. 1989. Rapid extraction of bacterial genomic DNA with guanidinium thiocyanate. Lett. Appl. Microbiol. 8:151-156.

23. Queiroz, D. M. M., G. A. Rocha, E. N. Mendes, A. P. Lage, A. C. T. Carvalgho, and A. J. A. Barbosa. 1990. A spiral microorganism in the stomach of pigs. Vet. Microbiol. 24:199-204.

24. Rappin, J. 1881. Contribution à l'étude de bacteries de la bouche à l'etat normal. (Quoted by R. S. Breed, E. G. D. Murray, and A. P. Hichens In Bergey's manual of determinative bacteriology (p. 217), 8th ed., Williams \&
Wilkins, Baltimore, 1974.)

25. Salomon, H. 1898. Über das Spirillum des Säugetiermagens und sein Verhalten zu den Belegzellen. Zentralbl. Bakteriol. Parasitenkd. Infektionskr. Hyg. Abt. 1 19:422-441.

26. Skirrow, M. B. 1994. Diseases due to Campylobacter, Helicobacter, and related bacteria. J. Comp. Pathol, 111:113-149.

27. Solnick, J. V., J. O'Rourke, A. Lee, B. Paster, F. E. Dewhirst, and L. S. Tompkins. 1993. An uncultured gastric spiral organism is a newly identified Helicobacter in humans. J. Infect. Dis. 168:379-385.

28. Stolte, M., E. Wellens, B. Bethke, M. Ritter, and H. Eidt. 1994. Helicobacter heilmannii (formerly Gastrospirillum hominis) gastritis: an infection transmitted by animals? Scan. J, Gastroenterol. 29:1061-1064.

29. Thompson, M. A., P. Storey, R. Greer, and G. J. Cleghorn. 1994. Canine human transmission of Gastrospirillum hominis. Lancet 343:1605-1607.

30. Ursing, J. B., H. Lior, and R. J. Owen. 1994. Proposal of minimal standards for describing new species of the family Campylobacteraceae. Int. J. Syst. Bacteriol. 44:842-845.

31. Weber, A. F., and E. F. Schmittdiel. 1962. Electron microscopic and bacteriologic studies of spirilla isolated from the fundic stomachs of cats and dogs. Am. J. Vet. Res. 23:422-427.

32. Wegmann, W., M. Aschwanden, N. Schaub, W. Aenishänslin, and K. Gyr. 1991. Gastrospirillum hominis-assozierte Gastritis-eine Zoonosis. Schweiz. Med. Wochenschr. 121:245-254. 